ISSN: 0212-5374

DOI: http://dx.doi.org/10.14201/et20143212352

\title{
CONOCIMIENTO, VALORACIÓN Y UTILIZACIÓN, POR PARTE DEL ALUMNADO, DE GOOGLE DRIVE COMO HERRAMIENTA DE TRABAJO COOPERATIVO
}

\author{
Knowledge, evaluation and use, by students, of Google \\ Drive as a tool for cooperative work
}

\section{Connaissance, évaluation et utilisation, pars les élèves, de Google Drive comme outil de travail coopératif}

\author{
Miguel Álvarez FerRón* y Luis SÁnChez CAÑIZARES*** \\ *Universidad de Murcia. Correo-e: malvarezferron@gmail.com; miguel.alvarez@ \\ um.es \\ *** IES Príncipe de Asturias. Lorca
}

Recibido: 13.11.2013; Aceptado: 28.03.2014; Publicado: 31.10.2014

BIBLID [0212-5374 (2014) 32, 2; 23-52]

Ref. Bibl. MIGUEL ÁLVAREZ FERRÓN y LUIS SÁNCHEZ CAÑIZARES. Conocimiento, valoración y utilización, por parte del alumnado, de Google Drive como herramienta de trabajo cooperativo. Enseñanza \& Teaching, 32, 2-2014, 23-52.

RESUMEN: La normativa que regula el sistema educativo español parece partir de un enfoque constructivista del proceso de enseñanza-aprendizaje. La metodología propia de este modelo pretende la construcción de aprendizaje por parte del alumnado que se interrelaciona en el aula, con la ayuda de un docente que ejerce la labor de guía. Es, por tanto, consustancial al mismo, el aprendizaje entre iguales en un contexto de interacción cooperativa.

Dentro de la tendencia generalizada a la aplicación de las Tecnologías de la Información y la Comunicación (TIC) en educación, se aprecia especial interés en la búsqueda de herramientas que faciliten el trabajo cooperativo en los centros escolares. 
MIGUEL ÁLVAREZ FERRÓN Y LUIS SÁNCHEZ CAÑIZARES

CONOCIMIENTO, VALORACIÓN Y UTILIZACIÓN, POR PARTE DEL ALUMNADO, DE GOOGLE DRIVE COMO HERRAMIENTA DE TRABAJO COOPERATIVO

En el plano teórico, han aparecido múltiples estudios sobre la materia. En el práctico, se han desarrollado numerosas aplicaciones basadas en la llamada web 2.0 que pretenden favorecer este tipo de aprendizaje.

Este trabajo describe la valoración general que una muestra de alumnos/as de un IES realiza sobre el trabajo cooperativo. También describe la frecuencia de uso de las TIC (y en concreto de la aplicación Google Drive como herramienta de trabajo cooperativo), la utilidad percibida y la influencia en la motivación del alumnado. En todos los casos, la valoración del alumnado fue claramente positiva.

Palabras clave: aprendizaje cooperativo, Google Drive, motivación, TIC, utilidad.

SUMMARY: The legislation governing the Spanish educational system, seems apparently focused on of a constructivist approach of the teaching-learning process. The methodology of this model aims to build students' learning through interaction in the classroom, with the help of a teacher who acts as a guide. It is, the refore, consustantial to it, the peer learning in a context of cooperative interaction.

Within the general trend towards the application of Information and communications technology (ICT) in education, there is special interest in the search for tools to facilitate collaborative learning in schools. At the theoretical level, there have been many studies on this subject. In practical terms, numerous applications have been developed based on the so-called Web 2.0 which try to encourage this type of learning.

This research describes the general assessment that a group of students from a secondary school performed on the cooperative work. It also describes the frequency of use of ICT (and specifically Google Drive application as a tool for cooperative work), perceived use fulness, and influence student motivation. In all cases, the assessment of students was clearly positive.

Key words: cooperative learning, Google Drive, motivation, ICT, utility.

RÉSUMÉ: La réglementation qui sert à la régulation du système éducatif espagnol part d'un point de vue constructif du processus de l'enseignement-apprentissage. La méthodologie de ce modèle prétend la construction de l'apprentissage des élèves qui interagissent dans la classe, avec l'aide d'un enseignant qui fait le travail de guide. Il est donc inhérente à lui-même, l'apprentissage entre pairs dans un contexte d'interaction coopérative.

Dans la tendance généralisée à la mise en oeuvre des Technologies de l'Information et de la Communication (TIC) dans l'enseignement, un intérêt particulier est apprécié dans la recherche d'outils pour faciliter le travail collaboratif dans les écoles. Au niveau théorique, il ya eu de nombreuses études à propos de ce sujet. Dans le projet pilote, de nombreuses applications ont été développées sur la base de Web 2.0 qui tentent d'encourager ce type d'apprentissage.

Ce document décrit l'évaluation globale qu'un groupe d'élèves d'un IEs realise sur le travail coopératif. Il décrit également la fréquence d'utilisation des Tic (et en 
MIGUEL ÁLVAREZ FERRÓN Y LUIS SÁNCHEZ CAÑIZARES

CONOCIMIENTO, VALORACIÓN Y UTILIZACIÓN, POR PARTE DEL ALUMNADO, DE GOOGLE DRIVE

COMO HERRAMIENTA DE TRABAJO COOPERATIVO

particulier l'application Google Drive comme un outil de travail coopératif), l'utilité perçue et l'influence sur la motivation des élèves. Dans tous les cas, l'évaluation des élèves est clairement positive.

Mots clé: pédagogie coopérative, Google Drive, motivation, TIC, utilité.

\section{INTRODUCCIÓN}

El presente estudio recoge un trabajo de investigación de naturaleza básica aplicada. En el mismo, por una parte, se exponen las prestaciones que, a pesar de sus limitaciones, facilitan las aplicaciones contenidas en Google Drive para realizar trabajo cooperativo en centros educativos. Por otra, se realiza un estudio del conocimiento y de la valoración que, de estas herramientas, realiza una muestra del alumnado del IES "Príncipe de Asturias" de Lorca, en el que se imparten enseñanzas de educación secundaria, bachillerato y formación profesional.

El trabajo se centra en Google Drive por dos motivos:

- Aunar en un único software la posibilidad de almacenar y compartir documentos y las aplicaciones ofimáticas que permiten trabajar sobre los mismos, limitándose otras aplicaciones como Dropbox a ofrecer el almacenamiento de archivos, permitiendo a los usuarios compartirlos.

- Una vez realizado el trabajo de campo, el porcentaje de sujetos encuestados que afirman utilizar aplicaciones distintas de Google Drive -en concreto el citado Dropbox- alcanza sólo el 0,96\% de la muestra.

No obstante, entendemos que muchas de las conclusiones que se obtienen y de las apreciaciones que se realizan son plenamente aplicables a otros servicios similares.

\section{OBjetivos}

Se fijan como objetivos primarios del presente trabajo de investigación los siguientes:

1. Determinar la frecuencia con que los alumnos del IES Príncipe de Asturias de Lorca realizan trabajos académicos del instituto colaborando en grupo.

2. Describir la valoración de la utilidad que el alumnado atribuye a la realización de trabajo cooperativo.

3. Determinar la frecuencia de uso de las TIC, por parte del alumnado, como herramientas para realizar trabajo cooperativo, prestando atención a los concretos dispositivos-aplicaciones utilizados.

4. Analizar la apreciación de alumnos y alumnas sobre la utilidad y la incidencia en la motivación derivada del uso de TIC en la realización de trabajos. 
MIGUEL ÁLVAREZ FERRÓN Y LUIS SÁNCHEZ CAÑIZARES

CONOCIMIENTO, VALORACIÓN Y UTILIZACIÓN, POR PARTE DEL ALUMNADO, DE GOOGLE DRIVE COMO HERRAMIENTA DE TRABAJO COOPERATIVO

5. Describir el nivel de conocimiento del alumnado sobre Google Drive, y las aplicaciones que lo integran, como herramienta tecnológica para desarrollar trabajo cooperativo.

6. Analizar la valoración de la utilidad de Google Drive como herramienta de trabajo cooperativo por parte de los alumnos que lo hayan utilizado, comparándolo con la apreciación individual de cada uno de ellos sobre la dificultad de su aprendizaje y recomendación de uso.

Son objetivos secundarios:

I. Calibrar la posible influencia de la disposición de acceso a Internet en el hogar en el uso de TIC en trabajo cooperativo.

II. Valorar la influencia de algunos aspectos como la nacionalidad del alumnado o el nivel educativo cursado en alguna de las variables objeto de estudio.

III. Determinar las preferencias del alumnado en el uso de TIC para la realización de trabajos académicos.

\section{MARCO TEÓRICO}

\subsection{Aprendizaje cooperativo}

\subsubsection{Concepto de aprendizaje cooperativo}

A la vista de lo contenido en los artículos 1 y 2 de La Ley Orgánica 2/2006, de 3 de mayo, de Educación, podría decirse que esta norma -reguladora de nuestro sistema educativo-, al igual que lo hacía la LOGSE, parece que pretende partir de un enfoque psicopedagógico y metodológico constructivista, al que -aunque desde puntos de vista muy diversos- realizaron aportaciones fundamentales autores como Piaget, Vygotski, Brunner o Ausubel. Esta visión del proceso enseñanzaaprendizaje considera al alumno constructor de su propio aprendizaje, y entiende que el papel que el profesor debe desempeñar es el de guía y orientador. En este contexto, la acción educativa debe incidir en lo que Vygotski llama "Zona de Desarrollo Próximo" que se establece viendo la diferencia entre lo que el alumno es capaz de hacer y de aprender por sí solo y lo que es capaz de hacer y aprender con el concurso de otras personas, observándolas, imitándolas, siguiendo sus instrucciones o colaborando con ellas. Este planteamiento va a transformar el aula en un lugar donde los alumnos van a realizar un "descubrimiento asistido" caracterizado por la colaboración entre iguales y la labor de guía asumida por el profesor.

Por esta relevancia otorgada al trabajo en equipo -en el que debe existir una adecuada interacción entre iguales- por el modelo curricular consagrado por la normativa legal, tal y como señala Úriz (1999), es fundamental contar con herramientas que posibiliten un trabajo en equipo que se ciña a los criterios pedagógicos del trabajo cooperativo. 
Johnson, Johnson y Holubec (1999: 5) consideran que «a cooperación consiste en trabajar juntos para alcanzar objetivos comunes". Esta afirmación conlleva la posibilidad de declarar que lo característico de una situación cooperativa es que los individuos que la integran persiguen obtener un resultado beneficioso tanto para sí mismos como para los demás miembros del grupo. Continúan estos autores exponiendo que "el aprendizaje cooperativo es el empleo didáctico de grupos reducidos en los que los alumnos trabajan juntos para maximizar su propio aprendizaje y el de los demás». Esta idea la enfatiza Pujolàs (2004: 81), al afirmar que trabajo cooperativo "no se trata sólo de hacer una misma cosa entre todos, sino también de hacer cada uno una cosa al servicio de una "comunidad" que persigue unas metas comunes". En fin, Fathman y Kessler (1993: 128) -citados por Trujillo y Ariza (2006: 12)- definen el aprendizaje cooperativo como el trabajo en grupo que se estructura cuidadosamente para que todos los estudiantes interactúen, intercambien información y puedan ser evaluados de forma individual por su trabajo".

\subsubsection{Componentes del aprendizaje cooperativo}

Johnson, Johnson y Holubec (1999) distinguen cinco elementos esenciales caracterizadores del aprendizaje cooperativo. Tales elementos, además, representan una disciplina que debe aplicarse rigurosamente para producir las condiciones que conduzcan a una acción cooperativa eficaz.

Se resumen en el siguiente cuadro:

\section{CUADRO 1}

Componentes del aprendizaje cooperativo

(basado en Johnson, Johnson y Holubec, 1999: 9)

\begin{tabular}{|l|l|}
\hline $\begin{array}{l}\text { - Interdependencia positiva (Debe ser claramente } \\
\text { percibida) }\end{array}$ & $\begin{array}{l}\text { - Responsabilidad individual y } \\
\text { grupal }\end{array}$ \\
\hline - Interacción promotora cara a cara & Evaluación grupal \\
\hline $\begin{array}{l}\text { - Aprendizaje de prácticas interpersonales } \\
\text { y grupales imprescindibles }\end{array}$ & \\
\hline
\end{tabular}

En resumen, existen múltiples estudios, referidos al aprendizaje cooperativo, que vienen a sustentar su idoneidad para el trabajo en el aula por cuanto:

- Incrementa la motivación y el rendimiento de los alumnos.

- Favorece la aparición de pensamiento crítico.

- Fomenta una mayor dedicación a la realización de tareas por parte del alumnado.

- Propicia un clima social adecuado y solidario, a la par que incide de manera positiva en la autoestima de cada alumno o alumna. 
MIGUEL ÁLVAREZ FERRÓN Y LUIS SÁNCHEZ CAÑIZARES

CONOCIMIENTO, VALORACIÓN Y UTILIZACIÓN, POR PARTE DEL ALUMNADO, DE GOOGLE DRIVE COMO HERRAMIENTA DE TRABAJO COOPERATIVO

\subsubsection{Aprendizaje cooperativo vs aprendizaje colaborativo}

Aunque no se trata de una cuestión que afecte a los objetivos del presente trabajo de investigación -bastando para este estudio la reafirmación de encontrarnos ante un supuesto de aprendizaje cooperativo y no colaborativo-, a la hora de tratar el marco teórico, parece aconsejable hacer referencia, aunque sea de una manera sucinta, a la diferencia existente entre aprendizaje cooperativo y aprendizaje colaborativo.

Tanto el aprendizaje cooperativo como el colaborativo tienen como fundamento teórico el modelo del constructivismo social de Vygotski, que considera el aprendizaje como una actividad eminentemente social. El aprendizaje cooperativo y el aprendizaje colaborativo pretenden que el conocimiento del alumnado surja y se incremente de la interacción entre iguales, bajo la guía del docente. Esta raíz común ha llevado a algunos autores, como Scagnoli (2005), a considerar que ambas expresiones son sinónimas.

Sin embargo, es mayoritaria la opinión doctrinal que considera que estos términos designan realidades diferentes. Así Suárez Guerrero (2010: 57) entiende que más que tratarse de dos términos que encierran una mera diferencia semántica "implica distinguir con el lenguaje dos hechos de interacción diferentes". Este autor cita en su obra a Panitz (1997), para el que

la colaboración es una filosofía de interacción donde los individuos son responsables de sus acciones, incluyendo el aprendizaje, y respetan las habilidades y contribuciones de sus compañeros. El aprendizaje colaborativo es una filosofía personal [...] comparte la autoridad y acepta la responsabilidad, entre todos, de las acciones del grupo. La premisa básica del aprendizaje colaborativo es la construcción del consenso a través de la cooperación de los miembros del grupo [...] El aprendizaje cooperativo es definido por un conjunto de procesos que ayudan a las personas a interactuar para lograr una meta específica [...] es más dirigido y muy controlado por el profesor.

En la siguiente tabla se resumen las principales características que diferencian aprendizaje cooperativo y aprendizaje colaborativo.

TABLA 1

Principales diferencias entre aprendizaje cooperativo y colaborativo

\begin{tabular}{|l|l|l|}
\cline { 2 - 3 } \multicolumn{1}{l|}{} & APRENDIZAJE COOPERATIVO & \multicolumn{1}{c|}{ APRENDIZAJE COLABORATIVO } \\
\hline Se considera: & Una técnica & Una filosofía \\
\hline Actividad se estructura: & De manera predefinida & De forma libre \\
\hline Presta mayor atención: & A la tarea & Al proceso \\
\hline Fomenta: & Interacción social & Creatividad \\
\hline Responsable del resultado: & El profesorado & El alumnado \\
\hline Dirigido a: & Grupos heterogéneos & Grupos con cierta preparación \\
\hline
\end{tabular}


MIGUEL ÁLVAREZ FERRÓN Y LUIS SÁNCHEZ CAÑIZARES

CONOCIMIENTO, VALORACIÓN Y UTILIZACIÓN, POR PARTE DEL ALUMNADO, DE GOOGLE DRIVE COMO HERRAMIENTA DE TRABAJO COOPERATIVO

\subsection{Web 2.0}

Al hablar de trabajo cooperativo y de TIC es fundamental abordar el concepto de Web 2.0. Este término, acuñado por Dougherty en el año 2004, se refiere a una generación de servicios basados en Internet que se caracterizan por poner el acento en la colaboración en línea, la interactividad y la posibilidad de que los usuarios, aunque no sean expertos, se conviertan también en contribuyentes, compartiendo contenidos que ellos mismos han creado. Según Anderson (2007: 6) -citado y traducido por Castañeda Quintero (ponencia sin paginar)-Web 2.0 "hace referencia a un grupo de tecnologías que facilitan la conexión social, y donde "todos y cada uno -de los usuarios se entiende- son capaces de añadir y editar la información"”.

El paso de un modelo de contenidos creados y colgados por unos pocos expertos - web 1.0 a otro, de marcado carácter social, que permite alumbrar contenidos de forma cooperativa, ha sido posible gracias al uso de tecnologías que facilitan tanto la creación por usuarios noveles como el flujo de estas creaciones a través de la Red. Entre las tecnologías propias de la web 2.0 se pueden destacar:

\section{CUADRO 2}

Principales tecnologías propias de la Web 2.0 (basado en O'Reilly, 2004)

\begin{tabular}{|l|l|}
\hline - Social networking & $\bullet$ Blogs \\
\hline - CMS (Sistemas de gestión de contenidos) & - Wikis \\
\hline - Organización social/inteligente información & $\bullet$ Podcasting \\
\hline - Almacenamiento web & $\bullet P 2 P$ \\
\hline
\end{tabular}

En atención a todo lo que se ha expuesto, es posible afirmar, siguiendo a Delgado Benito (2011), que las aplicaciones abiertas de la Web 2.0 son herramientas susceptibles de propiciar el trabajo cooperativo, permitiendo que el alumnado interactúe y se comunique, sobre su propio trabajo: con los docentes, con sus iguales y consigo mismo.

\subsection{Google Drive}

Es muy gráfica la metáfora empleada por Gutiérrez y López (2011, introducción), al referirse a Google como la "navaja suiza de Internet". Compartiendo en buena medida tal imagen, se puede afirmar que Google Drive es la herramienta de esa navaja helvética, pensada y desarrollada para servir como instrumento de trabajo cooperativo. De hecho, la propia compañía californiana la incluye dentro de su página web $^{1}$ dedicada a aplicaciones para uso educativo -Google Apps for Education - (todo ello contemplado con la debida cautela dada la naturaleza empresarial y los objetivos perseguidos por la compañía).

1. http://www.google.com/apps/intl/es/edu (consultado por última vez: 12/10/2013). 
MIGUEL ÁLVAREZ FERRÓN Y LUIS SÁNCHEZ CAÑIZARES

CONOCIMIENTO, VALORACIÓN Y UTILIZACIÓN, POR PARTE DEL ALUMNADO, DE GOOGLE DRIVE COMO HERRAMIENTA DE TRABAJO COOPERATIVO

Google Drive surge como tal el 24 de abril de 2012, englobando otros productos ya existentes como Google Documentos y Hojas de cálculo, Google Docs and Spreadsheets ${ }^{2}$. Este último producto es una suite de programas informáticos gratuitos que nació en el año 2005 y está inspirada por la filosofía propia de la Web 2.0. Sirve para crear y compartir documentos en línea, admitiendo la posibilidad de colaborar en grupo, trabajando sobre un mismo documento e, incluso, que este trabajo en colaboración se realice de manera simultánea.

El detalle de las concretas funciones y aplicaciones que incluye Google Drive, así como las utilidades y funcionalidades que aporta cada una de ellas, se detallan en la tabla siguiente:

TABLA 2

Aplicaciones integradas en Google Drive

\begin{tabular}{|c|c|}
\hline $\begin{array}{l}\text { FUNCIÓN/ } \\
\text { APLICACIÓN }\end{array}$ & FUNCIONALIDAD/UTILIDAD \\
\hline \multirow{6}{*}{$\begin{array}{l}\text { Almacenamiento } \\
\text { de archivos y } \\
\text { documentos }\end{array}$} & $\begin{array}{l}\text { Servicio de back up -copia de seguridad- remoto (en la nube) y } \\
\text { automático. }\end{array}$ \\
\hline & 5 GB de espacio gratuito a disposición del usuario. \\
\hline & $\begin{array}{l}\text { Posibilidad de sincronizar archivos con el ordenador o dispositivo móvil } \\
\text { del usuario, contando con control de versiones. }\end{array}$ \\
\hline & $\begin{array}{l}\text { Permite recuperar documentos de la papelera del PC (alta prestación e } \\
\text { innovación en materia de seguridad). }\end{array}$ \\
\hline & $\begin{array}{l}\text { Opción de subir o bajar archivos de forma masiva, respetando la } \\
\text { estructura de carpetas. }\end{array}$ \\
\hline & $\begin{array}{l}\text { Facultad de compartir carpetas o documentos, y de trabajar de manera } \\
\text { simultánea sobre los mismos, utilizando alguna de las aplicaciones de } \\
\text { la suite. }\end{array}$ \\
\hline \multirow{2}{*}{ Docs } & $\begin{array}{l}\text { Permite crear documentos de manera similar a cualquier otro procesador } \\
\text { de textos del mercado. }\end{array}$ \\
\hline & $\begin{array}{l}\text { Posibilita: creación y edición conjunta, sea o no simultánea, y compartir } \\
\text { documentos. }\end{array}$ \\
\hline \multirow[b]{2}{*}{ Hojas de cálculo } & Desarrolla funciones propias del software hoja de cálculo. \\
\hline & $\begin{array}{l}\text { Posibilita la creación y edición conjunta, sea o no simultánea, y compartir } \\
\text { trabajos. }\end{array}$ \\
\hline \multirow[b]{2}{*}{ Presentaciones } & Crea y edita diapositivas y presentaciones. \\
\hline & $\begin{array}{l}\text { Permite creación y edición conjunta, difusión vía web o compartiendo } \\
\text { creación. }\end{array}$ \\
\hline Formularios & $\begin{array}{l}\text { Posibilita crear encuestas en un editor de formularios y recopilar datos } \\
\text { en una hoja de cálculo que permite analizarlos y compartirlos. }\end{array}$ \\
\hline
\end{tabular}

2. Google Docs, hasta su integración en Google Drive, era una aplicación autónoma y como tal a ella se refieren los trabajos publicados sobre aprendizaje cooperativo utilizando herramientas de Google. 
MIGUEL ÁLVAREZ FERRÓN Y LUIS SÁNCHEZ CAÑIZARES

CONOCIMIENTO, VALORACIÓN Y UTILIZACIÓN, POR PARTE DEL ALUMNADO, DE GOOGLE DRIVE

COMO HERRAMIENTA DE TRABAJO COOPERATIVO

\begin{tabular}{|l|l|}
\hline \multicolumn{1}{|c|}{$\begin{array}{c}\text { FUNCIÓN/ } \\
\text { APLICACIÓN }\end{array}$} & \multicolumn{1}{|c|}{ FUNCIONALIDAD/UTILIDAD } \\
\hline Dibujos & $\begin{array}{l}\text { Permite crear elementos visuales, trabajar con ellos de forma colaborativa, } \\
\text { insertarlos en documentos o presentaciones y compartirlos. }\end{array}$ \\
\hline $\begin{array}{l}\text { Visualizador de } \\
\text { archivos }\end{array}$ & $\begin{array}{l}\text { Propicia que se puedan abrir diferentes tipos de archivos, en el navegador } \\
\text { web, aunque no se disponga del software necesario en el equipo } \\
\text { informático desde el que se accede. }\end{array}$ \\
\hline Buscador & $\begin{array}{l}\text { Buscador interno que permite encontrar las carpetas y documentos } \\
\text { almacenados. }\end{array}$ \\
\hline
\end{tabular}

Partiendo de esta información, así como de la contenida en el blog oficial de Google Drive y y de la recopilación realizada por Delgado Benito (2011: 332) se confeccionan dos tablas. La primera resume las características de Google Drive. La segunda recoge algunos fallos operativos de los que adolece.

TABLA 3

Principales características de Google Drive

\begin{tabular}{|c|c|}
\hline CARACTERÍSTICA & IMPLICACIÓN/ES \\
\hline Gratuidad & $\begin{array}{l}\text { La aplicación es totalmente gratuita, aunque con funcionalidades } \\
\text { limitadas, siendo las ampliaciones de pago. }\end{array}$ \\
\hline $\begin{array}{l}\text { Carácter } \\
\text { integrador }\end{array}$ & $\begin{array}{l}\text { Integra todas las herramientas -creación, edición, almacenamiento...- } \\
\text { en un solo producto. }\end{array}$ \\
\hline \multirow{2}{*}{$\begin{array}{l}\text { Funcionamiento } \\
\text { en línea }\end{array}$} & No requiere instalación previa de software. \\
\hline & Integración en la web. \\
\hline \multirow[b]{2}{*}{ Facilidad de uso } & Interfaz de usuario intuitiva. \\
\hline & $\begin{array}{l}\text { Existen automatismos para clasificación y actualización de carpetas y } \\
\text { documentos. }\end{array}$ \\
\hline $\begin{array}{l}\text { Facilidad de } \\
\text { edición }\end{array}$ & $\begin{array}{l}\text { Creación y edición de documentos o archivos muy similar -aunque más } \\
\text { limitada- a la de los programas ofimáticos tradicionales (v. g. Microsoft } \\
\text { Office). }\end{array}$ \\
\hline Disponibilidad & $\begin{array}{l}\text { Los archivos se almacenan en el servidor de Google y son fácilmente } \\
\text { accesibles/editables desde Internet. }\end{array}$ \\
\hline $\begin{array}{l}\text { Diseñado } \\
\text { para trabajo } \\
\text { cooperativo }\end{array}$ & $\begin{array}{l}\text { Permite trabajar a distintos usuarios de forma conjunta sobre un mismo } \\
\text { documento, ofreciendo la posibilidad de atribuir distintos roles y/o } \\
\text { privilegios. }\end{array}$ \\
\hline
\end{tabular}

TABLA 4

Limitaciones operativas de Google Drive

3. http://googledrive.blogspot.com.es/ (consultado por última vez el 6 de julio de 2013). 
MIGUEL ÁLVAREZ FERRÓN Y LUIS SÁNCHEZ CAÑIZARES

CONOCIMIENTO, VALORACIÓN Y UTILIZACIÓN, POR PARTE DEL ALUMNADO, DE GOOGLE DRIVE COMO HERRAMIENTA DE TRABAJO COOPERATIVO

\begin{tabular}{|l|l|}
\hline \multicolumn{1}{|c|}{ LIMITACIÓN } & \multicolumn{1}{c|}{ IMPLICACIÓN/ES } \\
\hline Menos funciones & $\begin{array}{l}\text { Programas más incompletos que los de paquetes ofimáticos } \\
\text { habituales. }\end{array}$ \\
\hline \multirow{3}{*}{ Seguridad } & $\begin{array}{l}\text { Aunque utiliza protocolos seguros, el hecho de ser una } \\
\text { herramienta online hace necesario adoptar una serie de } \\
\text { precauciones. }\end{array}$ \\
\cline { 2 - 2 } & $\begin{array}{l}\text { Necesaria diligencia de los usuarios en la gestión de sus } \\
\text { contraseñas. }\end{array}$ \\
\hline
\end{tabular}

A estas limitaciones que podríamos denominar "técnicas" a nivel usuario, que podrían resumirse en una funcionalidad limitada en la versión gratuita, hay que añadir una serie de inconvenientes, de mayor calado, que podría presentar el uso de este conjunto de aplicaciones como:

- Obligación de darse de alta como usuario de Google para poder utilizar esta aplicación.

- Sujeción a términos y condiciones de uso que suponen un contrato de adhesión en el que, además, Google se reserva el derecho de modificación unilateral.

- $\quad$ Es un hecho público y notorio que las políticas de privacidad y protección de datos de las grandes empresas de la comunicación, que Google encabeza, están siendo puestas en tela de juicio por numerosos estudios, organizaciones e instituciones.

\section{MATERIAL Y MÉTODOS}

\subsection{Introducción}

En los apartados precedentes se ha puesto en práctica una metodología exploratoria, consistente en una revisión bibliográfica, sobre Aprendizaje Cooperativo, Web 2.0 y Google Drive. Para abordar la discusión, que se desarrollará en el apartado 6, también se ha realizado una revisión bibliográfica de estudios sobre la aplicación de Google Docs como herramienta de trabajo cooperativo en educación.

La parte empírica del presente trabajo pretende cumplir los objetivos que se han enunciado en el apartado 2. Para ello se diseñó un estudio transversal, que incluye una parte descriptiva y otra analítica, realizadas entre los meses de febrero y julio de 2013. En los sucesivos epígrafes se describen los aspectos de diseño metodológico considerados y tenidos en cuenta en la investigación.

\subsection{Universo estudiado}


MIGUEL ÁLVAREZ FERRÓN Y LUIS SÁNCHEZ CAÑIZARES

CONOCIMIENTO, VALORACIÓN Y UTILIZACIÓN, POR PARTE DEL ALUMNADO, DE GOOGLE DRIVE

COMO HERRAMIENTA DE TRABAJO COOPERATIVO

La población objeto de estudio está compuesta por el alumnado del IES Príncipe de Asturias. En este centro, ubicado en la localidad murciana de Lorca, se imparten las siguientes enseñanzas: Educación Secundaria Obligatoria; Bachillerato; dos Programas de Cualificación Profesional Inicial -Auxiliar dependiente de comercio y almacén y Auxiliar de oficina; -un Ciclo Formativo de Grado Medio -Comercio- y un Ciclo Formativo de Grado Superior: Comercio Internacional.

Además, existe un grupo de 1. de ESO ( 25 alumnos) que trabaja a través de métodos cooperativos de aprendizaje, utilizando Google Drive como herramienta a través de la cual se articula el trabajo cooperativo.

\subsection{Método de investigación}

En atención al contexto en el que se ha realizado el estudio, así como a los objetivos que se persiguen en el mismo, se ha optado por la utilización del cuestionario como método. El mismo consta de 20 preguntas cerradas de las cuales:

- Las cuatro primeras son de identificación (sexo, nacionalidad, edad y nivel educativo del encuestado).

- Tres son dicotómicas, dos referidas a cuestiones de hecho y la otra de información.

- Tres son cerradas y admiten más de una respuesta.

- Las diez restantes son cerradas y admiten una única respuesta.

En la elaboración del mismo se han seguido las directrices señaladas por Cadoche y recogidas por Díaz y Piñana (2013).

\subsection{Determinación de la muestra necesaria}

Para determinar la muestra necesaria, se ha utilizado la calculadora en línea para encuestas contenida en la página web de Netquest ${ }^{4}$. En el cálculo se han respetado los valores estándar de los siguientes aspectos: margen de error, 5\%; nivel de confianza, 95\%; heterogeneidad de la muestra, 50\%.

Para lograr una muestra representativa se ha optado por calcular su tamaño estratificándola: dividiendo la población en distintos grupos para su estudio. Dicha división supone un incremento de la muestra total necesaria, pero redunda en beneficio de la fiabilidad del estudio y asegura la representatividad de la muestra en aquellos casos en los que alguna variable se quiera analizar en función del nivel académico. Los grupos estudiados y la muestra recomendada para cada uno de ellos, que se han obtenido al utilizar la calculadora, se detallan en la Tabla 5.

4. Www.netquest.com, Netquest es una empresa especializada en la recolección y procesamiento de datos en línea. 
MIGUEL ÁLVAREZ FERRÓN Y LUIS SÁNCHEZ CAÑIZARES

CONOCIMIENTO, VALORACIÓN Y UTILIZACIÓN, POR PARTE DEL ALUMNADO, DE GOOGLE DRIVE COMO HERRAMIENTA DE TRABAJO COOPERATIVO

A fin de asegurar la fiabilidad de los cálculos se han comparado los valores obtenidos con la tabla estadística recogida por Díaz y Piñana (2013: 13).

TABLA 5

Muestra necesaria para cada grupo estudiado (calculadora Netquest)

\begin{tabular}{|c|c|c|}
\hline GRUPO ESTUDIADO & TOTAL ALUMNOS/AS & MUESTRA RECOMENDADA \\
\hline 1.-2.. ESO & 365 & 188 \\
\hline $3 .{ }^{\circ}-4 .{ }^{\circ}$ ESO & 250 & 152 \\
\hline Bachillerato & 253 & 153 \\
\hline PCPI & 44 & 40 \\
\hline CFGM & 40 & 37 \\
\hline CFGS & 29 & 28 \\
\hline Grupo cooperativo & 25 & 24 \\
\hline
\end{tabular}

TABLA 6

Tabla estadística para determinación de muestras (Díaz y Piña)

\begin{tabular}{|c|c|c|c|c|c|c|}
\hline \multirow{2}{*}{ Población } & \multicolumn{4}{|c|}{ Margen de error } & \multicolumn{2}{c|}{ Intervalo de confianza } \\
\cline { 2 - 7 } & $\mathbf{1 0 \%}$ & $\mathbf{5 \%}$ & $\mathbf{1 \%}$ & $\mathbf{9 0 \%}$ & $\mathbf{9 5 \%}$ & $\mathbf{9 9 \%}$ \\
\hline $\mathbf{1 0 0}$ & 50 & 80 & 99 & 74 & 80 & 88 \\
\hline $\mathbf{5 0 0}$ & 81 & 218 & 476 & 176 & 218 & 286 \\
\hline $\mathbf{1 . 0 0 0}$ & 88 & 278 & 906 & 215 & 278 & 400 \\
\hline $\mathbf{1 0 . 0 0 0}$ & 96 & 370 & 4.900 & 264 & 370 & 623 \\
\hline $\mathbf{1 0 0 . 0 0 0}$ & 96 & 383 & 8.763 & 270 & 383 & 660 \\
\hline $\mathbf{1 . 0 0 0 . 0 0 0}$ & 97 & 384 & 9.513 & 271 & 384 & 664 \\
\hline
\end{tabular}


MIGUEL ÁLVAREZ FERRÓN Y LUIS SÁNCHEZ CAÑIZARES

CONOCIMIENTO, VALORACIÓN Y UTILIZACIÓN, POR PARTE DEL ALUMNADO, DE GOOGLE DRIVE COMO HERRAMIENTA DE TRABAJO COOPERATIVO

Aplicando la misma, realizando los cálculos oportunos, el tamaño de la muestra recomendada sería el siguiente:

TABLA 7

Muestra necesaria para cada grupo estudiado

\begin{tabular}{|c|c|c|}
\hline GRUPO ESTUDIADO & TOTAL ALUMNOS/AS & MUESTRA RECOMENDADA \\
\hline $1 .^{\circ}-2 .{ }^{\circ}$ ESO & 365 & 159 \\
\hline $3 .^{\circ}-4 .^{\circ}$ ESO & 250 & 109 \\
\hline Bachillerato & 253 & 110 \\
\hline PCPI & 44 & 36 \\
\hline CFGM & 40 & 32 \\
\hline CFGS & 29 & 23 \\
\hline Grupo cOoperativo & 25 & 20 \\
\hline
\end{tabular}

Por ser mayor el valor de la muestra recomendada obtenido utilizando la calculadora de Netquest, se ha optado por utilizar éste para garantizar al máximo la representatividad de la muestra estudiada.

Se trata de una muestra no probabilística por cuanto la elección de los elementos no ha dependido de la probabilidad, sino de las características de la investigación, en la que se ha estado a merced de la disponibilidad y colaboración voluntaria del alumnado.

\subsection{Trabajo de campo}

El cuestionario se elaboró con la aplicación Formularios incluida en Google Drive. El cuestionario estuvo a disposición del alumnado, al que se facilitó un enlace web, de forma que, los que lo deseasen, pudiesen cumplimentarlo. El formulario estuvo disponible entre el 6 de mayo y el 16 de junio de 2013, fecha en la que se cerró el acceso al mismo.

Para aquellos grupos en los que el número de cuestionarios necesarios suponía casi el 100\% de los alumnos y alumnas -PCPI, CFGM, CFGS y Grupo de Aprendizaje Cooperativo- se realizó una sesión explicativa por parte de los distintos tutores.

\subsection{Recopilación y análisis de los datos obtenidos}

Como se ha adelantado en el epígrafe anterior, los datos se recogieron en línea a través de la herramienta Formularios contenida en Google Drive. Para asegurar la integridad y homogeneidad se marcaron todas las preguntas del cuestionario con la opción "respuesta obligatoria", lo que impide al entrevistado enviar el formulario hasta que se hayan contestado todas las preguntas planteadas.

La herramienta descarga automáticamente las respuestas al cuestionario en una hoja de cálculo. No obstante, debido a las menores prestaciones de la hoja de 
MIGUEL ÁLVAREZ FERRÓN Y LUIS SÁNCHEZ CAÑIZARES

CONOCIMIENTO, VALORACIÓN Y UTILIZACIÓN, POR PARTE DEL ALUMNADO, DE GOOGLE DRIVE COMO HERRAMIENTA DE TRABAJO COOPERATIVO

cálculo integrada en Google Drive -a las que se ha hecho referencia en este trabajo- para la recopilación y análisis de datos se optó por exportar éstos a Microsoft Excel. La versión de Excel utilizada ha sido la de 2010.

\section{Resultados}

\subsection{Introducción}

Aplicando la metodología empleada en el presente estudio, a la que se ha hecho referencia en el apartado anterior, se procede a la descripción y análisis de los datos obtenidos, para dar respuesta a las cuestiones planteadas en los objetivos que persigue esta investigación.

\subsection{Descripción y caracterización de la muestra}

En la muestra total, que comprende a 622 estudiantes, un 67,04\% (417) son mujeres, mientras que el 32,16\% restante (205) son hombres. En cuanto a la proporción entre alumnado español y alumnado extranjero, el primero constituye el $79,58 \%$ de la muestra, mientras el segundo alcanza el 20,42\% de la misma. Estos datos se concretan en la siguiente tabla:

TABLA 8

Distribución por sexos y procedencia geográfica en la muestra

\begin{tabular}{|c|c|c|c|c|c|}
\hline & ESPAÑOLES & RESTO EUROPA & SUDAMÉRICA & MAGREB & RESTO ÁFRICA \\
\hline Hombres & 164 & 7 & 28 & 5 & 1 \\
\hline Mujeres & 331 & 8 & 44 & 29 & 5 \\
\hline Total & 495 & 15 & 72 & 34 & 6 \\
\hline
\end{tabular}

Atendiendo a los niveles académicos, el número de cuestionarios estudiados en cada uno de ellos se ajusta a los datos obtenidos tras el cálculo de la muestra necesaria, consignado anteriormente en la Tabla 6.

Por considerarlo útil, como dato a tener en cuenta en el análisis de otras variables, se ha descrito el número de alumnos/as que disponen de conexión a Internet en su casa y los que no, obteniendo los resultados que se contienen en el Gráfico 1. 
MIGUEL ÁLVAREZ FERRÓN Y LUIS SÁNCHEZ CAÑIZARES

CONOCIMIENTO, VALORACIÓN Y UTILIZACIÓN, POR PARTE DEL ALUMNADO, DE GOOGLE DRIVE COMO HERRAMIENTA DE TRABAJO COOPERATIVO

GRÁFICO 1

Porcentaje de alumnado que dispone de conexión a Internet en casa

\begin{tabular}{|c|c|c|c|}
\hline & \\
\hline \\
$50,00 \%$ \\
$30,00 \%$ \\
$20,00 \%$ \\
$10,00 \%$ \\
$0,00 \%$
\end{tabular}

\subsection{Descripción de la frecuencia con la que realizan trabajo colaborando}

De los 622 alumnos y alumnas que componen la muestra sólo el 1,77\% responden que no realizan trabajos o tareas del instituto cooperando con sus compañeros. No obstante, el 63,44\% de las respuestas negativas -siete de once-son incoherentes por cuanto en las preguntas del cuestionario referentes al trabajo en equipo responden como si habitualmente trabajasen en equipo. Dado que las preguntas sucesivas son más complejas y, además, existe coherencia entre las mismas, se entiende que en estos siete casos los sujetos encuestados han incurrido en un error. Puesto que la pregunta en la que se ha manifestado la incidencia plantea una cuestión dicotómica se procede como si la respuesta hubiese sido afirmativa.

Una vez realizada esta operación, se obtiene como resultado que frente $a$ un 99,36\% de sujetos que dicen trabajar cooperando con sus compañeros/as, sólo un $0,64 \%$ del alumnado encuestado manifiesta no realizar tareas académicas trabajando en equipo con sus compañeros.

Parece importante destacar que, dentro de ese 0,64\%, el 75\% cursan un PCPI y son hombres. 
MIGUEL ÁLVAREZ FERRÓN Y LUIS SÁNCHEZ CAÑIZARES

CONOCIMIENTO, VALORACIÓN Y UTILIZACIÓN, POR PARTE DEL ALUMNADO, DE GOOGLE DRIVE COMO HERRAMIENTA DE TRABAJO COOPERATIVO

\subsection{Descripción de la valoración del trabajo en equipo}

\section{GRÁFICO 2}

Percepción de la utilidad de trabajo cooperando

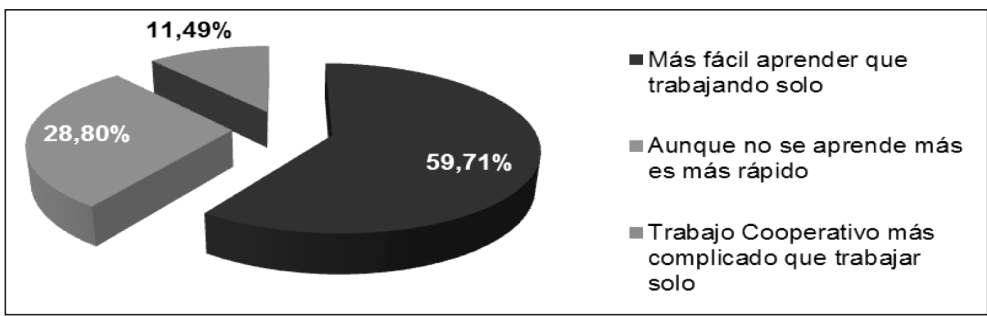

Tal y como se puede apreciar en el Gráfico 2 sólo el 11,49\% de los alumnos que manifestaron realizar tareas en equipo -71 de 618- valora de forma negativa esa manera de trabajar.

El 88,51\% restante, por el contrario, afirma percibir el trabajo en equipo como útil, con el matiz que se puede observar en el Gráfico 1:

- $\quad$ El 28,80\% del total, 178 de 618, valoran la utilidad del trabajo cooperando con sus compañeros sólo en la medida en que favorece que el trabajo se realice más rápido, considerando que esa forma de trabajar no redunda en beneficio del propio aprendizaje.

- $\quad$ El 59,71\% del total, por su parte, considera útil el trabajo cooperando con sus compañeros, y manifiesta que trabajar en equipo facilita el aprendizaje.

5.5. Descripción de la frecuencia del uso de TIC para realizar trabajos cooperando $y$ de los dispositivos de acceso a Internet utilizados

GRÁFICO 3

Uso NTIC para realizar trabajos en equipo

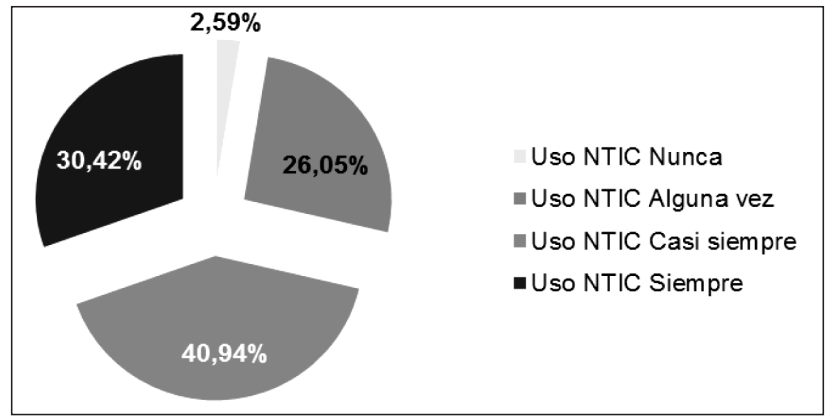


MIGUEL ÁLVAREZ FERRÓN Y LUIS SÁNCHEZ CAÑIZARES

CONOCIMIENTO, VALORACIÓN Y UTILIZACIÓN, POR PARTE DEL ALUMNADO, DE GOOGLE DRIVE

COMO HERRAMIENTA DE TRABAJO COOPERATIVO

En el Gráfico 3 aparece reflejada la frecuencia con la que el alumnado manifiesta utilizar las TIC para realizar trabajos con sus compañeros. Como se puede apreciar la frecuencia de uso puede ser calificada como alta puesto que la suma de los que manifiestan no utilizar nunca las TIC con los que aseguran realizar un uso eventual no alcanza el 30\% del total de la muestra.

En cuanto a los dispositivos que el alumnado dice utilizar preferentemente para acceder a Internet se reflejan en la Tabla 9.

TABLA 9

Dispositivos usados para acceder a Internet

\begin{tabular}{|l|c|c|c|c|}
\hline & \multicolumn{2}{|c|}{ ALM. ESPAÑOL } & \multicolumn{2}{c|}{ ALM. EXTRANJERO } \\
\hline Dispositivo (s) & N.. & $\%$ & N.o & $\%$ \\
\hline Ordenador & 141 & 28,48 & 40 & 31,50 \\
\hline Ordenador, PPC, tableta y teléfono & 11 & 2.22 & & \\
\hline Ordenador, PPC y teléfono & 8 & 1,62 & & \\
\hline Ordenador y tableta & 12 & 2,42 & & \\
\hline Ordenador, tableta y teléfono & 53 & 10,71 & 13 & 10,24 \\
\hline Ordenador y teléfono & 201 & 40,61 & 50 & 39,37 \\
\hline PPC y teléfono & 3 & 0,61 & & \\
\hline Teléfono & 64 & 12,93 & 24 & 18,90 \\
\hline Ordenador, IPOd & 1 & 0,20 & & \\
\hline Ordenador, PPC, tableta, teléfono, PSP3 & 1 & 0,20 & & \\
\hline
\end{tabular}

1.․ Un $14,15 \%$ del alumnado manifiesta que el dispositivo que utilizan con mayor frecuencia para acceder a Internet es el teléfono móvil -cuyo uso está prohibido en muchos institutos-.

2. - Ese porcentaje se alcanza cuando el teléfono es considerado en solitario, disparándose su uso, hasta un 69,26\%, cuando se combina su utilización con la de otros dispositivos.

3.․ La opción que manifiesta utilizar la mayoría de los encuestados es la que aglutina ordenador personal y teléfono móvil.

Además de todo lo que se ha señalado, también se considera importante hacer referencia a las diferencias existentes en cuanto al uso de dispositivos en función de lo expresado por el alumnado español y por el extranjero, por cuanto:

- El alumnado extranjero manifiesta utilizar un menor número de dispositivos que el nacional.

- $\quad$ No se aprecia una diferencia relevante en el porcentaje de uso atribuido a cada dispositivo atendiendo al origen del alumnado, limitándose las diferencias existentes al uso por parte del alumnado nacional de un mayor número de dispositivos. 
En el Gráfico 4 se analiza la posible incidencia del hecho de que el alumno disponga, o no, de conexión a Internet en su casa, en el uso de TIC para realizar trabajos cooperando. Tras el análisis de las respuestas facilitadas por los estudiantes, se puede concluir que el hecho de no disponer de Internet en casa influye negativamente en el uso de TIC por parte del alumnado.

\section{GRÁFICO 4}

Uso NTIC para realizar trabajos cooperando

en función de si el alumnado dispone de conexión a Internet en su casa

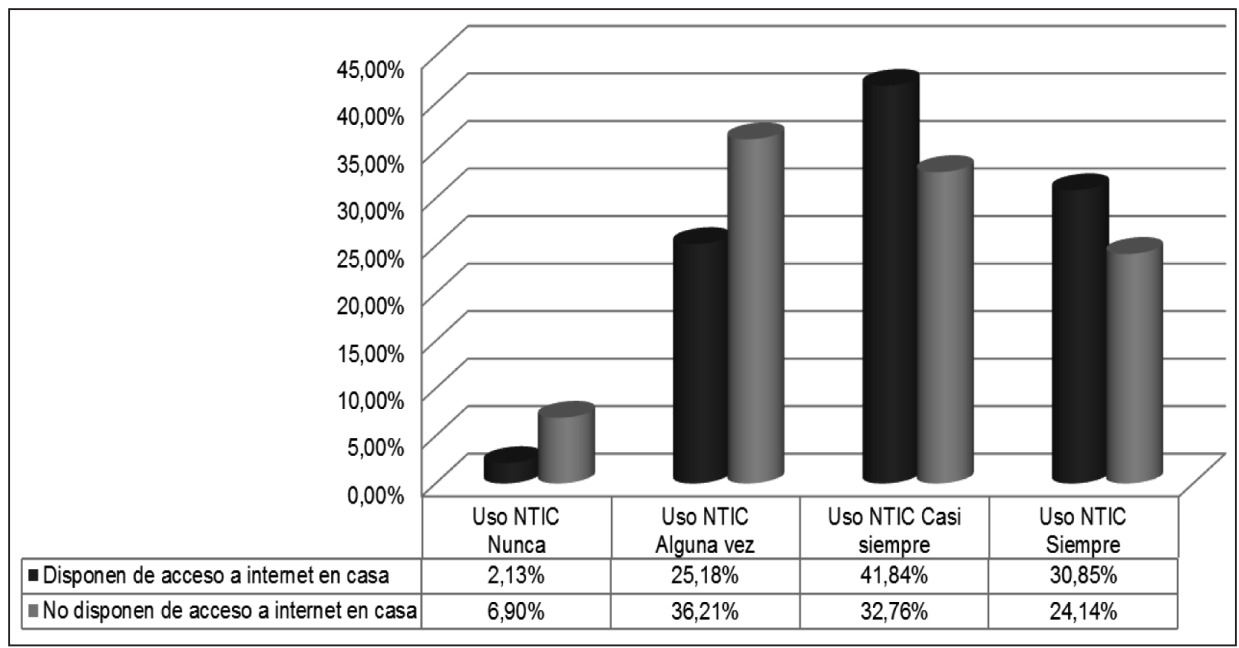

Lo que no permite el análisis es conocer si esa influencia se deriva del mero no disponer de medios materiales, o de un menor dominio de los mismos, pese a disponer de ellos en el Instituto.

Los más destacados entre los concretos medios TIC utilizados por los alumnos y alumnas para trabajar cooperando con sus compañeros y compañeras se consignan en la Tabla 10. 
MIGUEL ÁLVAREZ FERRÓN Y LUIS SÁNCHEZ CAÑIZARES

CONOCIMIENTO, VALORACIÓN Y UTILIZACIÓN, POR PARTE DEL ALUMNADO, DE GOOGLE DRIVE COMO HERRAMIENTA DE TRABAJO COOPERATIVO

TABLA 10

Detalle del uso de NTIC por el alumnado para trabajar con sus compañeros/as

\begin{tabular}{|l|c|c|}
\hline \multicolumn{1}{|c|}{ NTIC } & $\mathrm{N}^{\circ}$ & $\%$ \\
\hline $\begin{array}{l}\text { Redes Sociales (Facebook, Twitter, Tuenti u otra/s), Correo electrónico., } \\
\text { Mensajeria instantánea (Whatsapp u otro/s) }\end{array}$ & 82 & $13,18 \%$ \\
\hline $\begin{array}{l}\text { Google Drive., Redes sociales (Facebook, Twitter, Tuenti u otra/s), } \\
\text { Correo electrónico., Mensajería instantánea (Whatsapp u otro/s) }\end{array}$ & 46 & $7,40 \%$ \\
\hline $\begin{array}{l}\text { Google Drive., Redes sociales (Facebook, Twitter, Tuenti u otra/s), } \\
\text { Mensajeria instantánea (Whatsapp u otro/s) }\end{array}$ & 40 & $6,43 \%$ \\
\hline $\begin{array}{l}\text { Redes sociales (Facebook, Twitter, Tuenti u otra/s), Mensajería } \\
\text { instantánea (Whatsapp u otro/s) }\end{array}$ & 37 & $5,95 \%$ \\
\hline Google Drive., Wikis & 16 & $5,57 \%$ \\
\hline Redes sociales (Facebook, Twitter, Tuenti u otra/s), Correo electrónico. & 25 & $4,02 \%$ \\
\hline Wikis y Blogs Compartidos & 24 & $3,86 \%$ \\
\hline Correo electrónico & 23 & $3,70 \%$ \\
\hline $\begin{array}{l}\text { Google Drive., Redes sociales (Facebook, Twitter, Tuenti u otra/s), } \\
\text { Correo electrónico. }\end{array}$ & 19 & $3,05 \%$ \\
\hline $\begin{array}{l}\text { Google Drive., Redes sociales (Facebook, Twitter, Tuenti u otra/s), } \\
\text { Correo electrónico., Mensajeria instantánea (Whatsapp u otro/s), Blogs } \\
\text { compartidos }\end{array}$ & 19 & $3,05 \%$ \\
\hline $\begin{array}{l}\text { Redes sociales (Facebook, Twitter, Tuenti u otra/s), Correo electrónico., } \\
\text { Mensajeria instantánea (Whatsapp u otro/s), Blogs compartidos }\end{array}$ & 18 & $2,89 \%$ \\
\hline Wikis & 18 & $2,89 \%$ \\
\hline Redes sociales (Facebook, Twitter, Tuenti u otra/s) & 17 & $2,73 \%$ \\
\hline Google Drive, correo electrónico, wikis y blogs compartidos & 14 & $2,25 \%$ \\
\hline $\begin{array}{l}\text { Redes sociales (Facebook, Twitter, Tuenti u otra/s), Correo electrónico., } \\
\text { Mensajeria instantánea (Whatsapp u otro/s), Wikis }\end{array}$ & 14 & $2,25 \%$ \\
\hline $\begin{array}{l}\text { Redes sociales (Facebook, Twitter, Tuenti u otra/s), Correo electrónico., } \\
\text { Mensajería instantánea (Whatsapp u otro/s), Wikis, Blogs compartidos }\end{array}$ & 14 & $2,25 \%$ \\
\hline Correo electrónico y mensajería instantánea & 13 & $2,09 \%$ \\
\hline Google Drive & 12 & $1,93 \%$ \\
\hline Google Drive, correo electrónico y mensajería instantánea & 12 & $1,93 \%$ \\
\hline Google Drive, correo electrónico y wikis & $1,93 \%$ \\
\hline Mensajeria instantánea (Whatsapp u otro/s) & $1,93 \%$ \\
\hline
\end{tabular}


MIGUEL ÁLVAREZ FERRÓN Y LUIS SÁNCHEZ CAÑIZARES

CONOCIMIENTO, VALORACIÓN Y UTILIZACIÓN, POR PARTE DEL ALUMNADO, DE GOOGLE DRIVE COMO HERRAMIENTA DE TRABAJO COOPERATIVO

5.6. Análisis de la opinión sobre utilidad del uso de TIC en trabajo cooperativo

GRÁFICO 5

Opinión sobre la utilidad de uso de NTIC para el trabajo cooperando

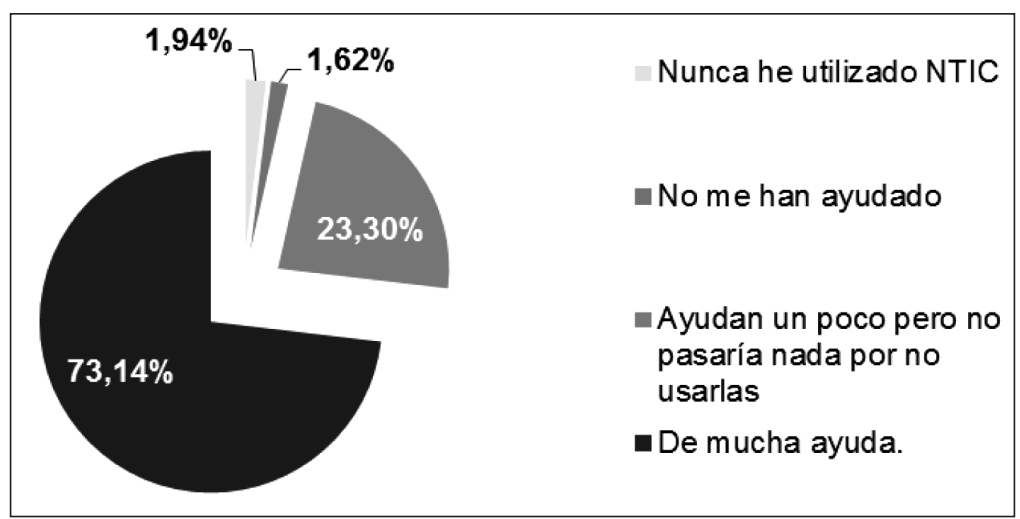

El Gráfico 5 describe como una amplia mayoría de la muestra considera que las TIC resultan de mucha ayuda a la hora de trabajar cooperando.

Es importante destacar que los valores extremos en lo que a apreciación de utilidad de las Tic se refiere se encuentran en los Ciclos Formativos. Así, el que más valora la utilidad de las TIC es el alumnado del Ciclo de Grado Superior, donde se alcanza un 100\% de respuestas que consideran las TIC de mucha ayuda.

Por el contrario, los alumnos y alumnas del Ciclo de Grado Medio, aunque no manifiestan que consideran poco útiles las Tic, son los que las valoran como útiles en un porcentaje inferior.

TABLA 11

Descripción utilidad Tic por grupos

\begin{tabular}{|l|c|c|c|c|c|c|c|}
\hline & $\begin{array}{c}1 .-2 .{ }^{\circ} \\
\text { ESO }\end{array}$ & $\begin{array}{c}3 .{ }^{\circ}-4 .{ }^{\circ} \\
\text { ESO }\end{array}$ & BAC & PCPI & CFGM & CFGS & COOP \\
\hline Nunca uso TIC & $2,79 \%$ & $1,97 \%$ & - & $12,50 \%$ & $2,86 \%$ & - & - \\
\hline No me han ayudado & $2,97 \%$ & - & - & - & - & - & - \\
\hline Ayudan un poco $[\ldots]$ & $15,08 \%$ & $18,42 \%$ & $37,25 \%$ & $22,50 \%$ & $45,71 \%$ & - & $12,50 \%$ \\
\hline De mucha ayuda & $79,33 \%$ & $79,61 \%$ & $62,75 \%$ & $70,00 \%$ & $51,43 \%$ & $100 \%$ & $87,50 \%$ \\
\hline
\end{tabular}


MIGUEL ÁLVAREZ FERRÓN Y LUIS SÁNCHEZ CAÑIZARES

CONOCIMIENTO, VALORACIÓN Y UTILIZACIÓN, POR PARTE DEL ALUMNADO, DE GOOGLE DRIVE COMO HERRAMIENTA DE TRABAJO COOPERATIVO

GRÁFICO 6

Análisis del uso de NTIC relacionado con la consideración de su utilidad

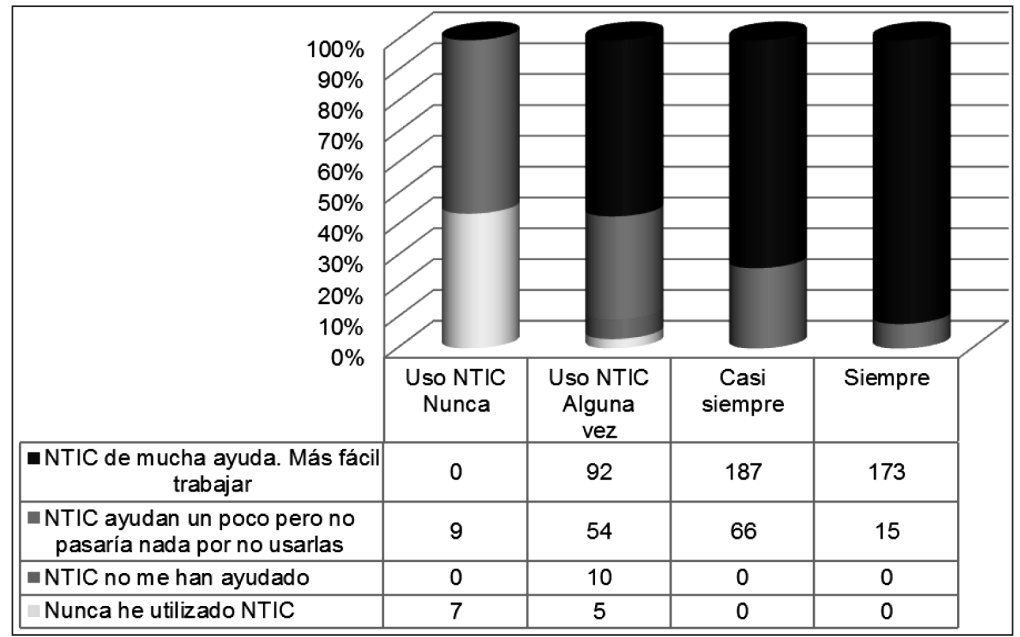

Resulta muy interesante analizar la opinión sobre la utilidad del uso de TIC relacionando esta variable con el uso efectivo de las TIC para trabajar en equipo. El resultado de este análisis se puede apreciar en el Gráfico 6, del que se concluye que existe una relación entre la valoración positiva de la utilidad de las TIC y el uso que, de las mismas, manifiesta hacer el alumnado. De hecho, en el caso de mayor utilización de las Tic, la valoración positiva de las mismas llega al 92\% de la muestra, correspondiendo el 8\% restante a la opinión indiferente y no existiendo ningún sujeto que no considere útil el uso de las Tic.

\subsection{Análisis de la opinión sobre influencia del uso de TIC en la motivación}

GRÁFICO 7

Influencia del uso de NTIC en la motivación

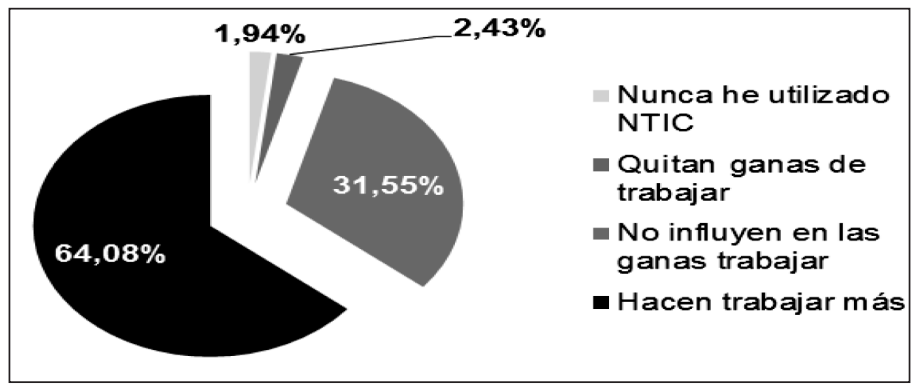


En lo referente a la valoración sobre la influencia del uso de Tic en la motivación, el alumnado considera, de forma mayoritaria, que tal influencia existe y es positiva; el porcentaje de la muestra que considera que las TIC influyen de forma negativa en su motivación no llega al 3\%.

Si se observa este dato en función de los grupos llegamos a la paradójica conclusión de que el grupo que manifiesta valorar más la utilidad del uso de las TIC, alumnos y alumnas del CFGS, es el único que no considera que éstas influyan positivamente en la motivación: el 58,33\% piensa que el uso de TiC no afecta a la motivación, frente a un $41,67 \%$ que cree que son positivas. El grupo que estima en mayor medida que el uso de TIC influye de forma positiva en la motivación es el de Aprendizaje Cooperativo, en el que un 95,83\% manifiesta esta opinión. El único grupo en el que se encuadran individuos que consideran que la influencia de las TIC en la motivación es negativa es el de 1.ํ-․ํำ de ESO.

TABLA 12

Descripción influencia en la motivación TIC por grupos

\begin{tabular}{|l|c|c|c|c|c|c|c|}
\hline & $\begin{array}{c}1 .{ }^{\circ}-2 . .^{\circ} \\
\text { ESO }\end{array}$ & $\begin{array}{c}3 .{ }^{\circ}-4 .{ }^{\circ} \\
\text { ESO }\end{array}$ & BAC & PCPI & CFGM & CFGS & COOP \\
\hline Nunca he usado TIC & $2,79 \%$ & $1,97 \%$ & - & $7,50 \%$ & $2,86 \%$ & - & - \\
\hline Influencia negativa & $5,99 \%$ & - & - & - & - & - & - \\
\hline No influyen & $15,08 \%$ & $41,45 \%$ & $41,83 \%$ & $37,50 \%$ & $31,43 \%$ & $58,33 \%$ & $4,17 \%$ \\
\hline Hacen trabajar más & $76,54 \%$ & $56,58 \%$ & $58,17 \%$ & $55,00 \%$ & $65,71 \%$ & $41,67 \%$ & $95,83 \%$ \\
\hline
\end{tabular}

En el Gráfico 8 se puede apreciar como, al analizar de manera conjunta el uso de las TIC y la consideración de su influencia en la motivación, la relación que se establece entre ambas variables es directamente proporcional: a mayor uso de TIC se tiende a considerar de manera más positiva su influencia en la motivación.

Como colofón a los dos epígrafes que acaban de ser tratados, en el Gráfico 9 se analizan, de manera conjunta: la percepción de utilidad y la influencia en la motivación de las Tic. La conclusión que se extrae es la existencia de una relación de proporción directa entre ambas variables. 
MIGUEL ÁLVAREZ FERRÓN Y LUIS SÁNCHEZ CAÑIZARES

CONOCIMIENTO, VALORACIÓN Y UTILIZACIÓN, POR PARTE DEL ALUMNADO, DE GOOGLE DRIVE COMO HERRAMIENTA DE TRABAJO COOPERATIVO

GRÁFICO 8

Comparación del uso de NTIC y percepción de la motivación

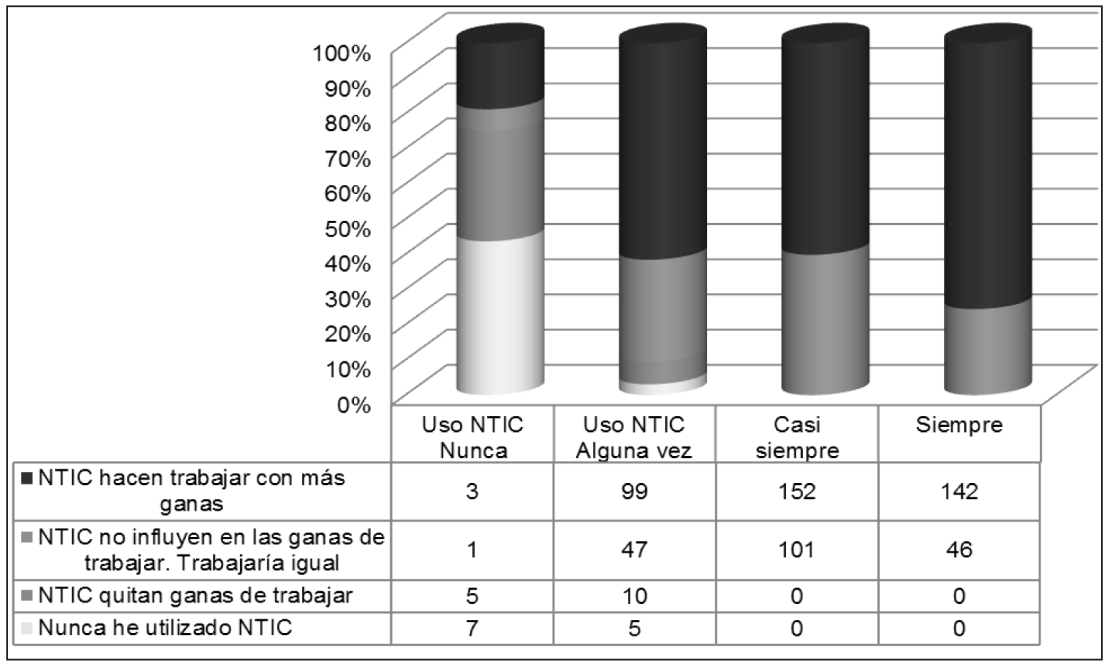

GRÁFICO 9

Comparación percepción de utilidad y motivación NTIC

\begin{tabular}{|c|c|c|c|c|}
\hline & \\
\hline & & & \\
\hline
\end{tabular}


MIGUEL ÁLVAREZ FERRÓN Y LUIS SÁNCHEZ CAÑIZARES

CONOCIMIENTO, VALORACIÓN Y UTILIZACIÓN, POR PARTE DEL ALUMNADO, DE GOOGLE DRIVE COMO HERRAMIENTA DE TRABAJO COOPERATIVO

\subsection{Conocimiento y uso de Google Drive por parte del alumnado}

El 48,39\% de la muestra total manifiesta conocer Google Drive. Entre ellos:

- Un 10,59\% no lo ha utilizado nunca.

- Un 29,60\% lo utiliza puntualmente.

- Un $25,23 \%$ lo utiliza de vez en cuando.

- Un 34,53\% lo utiliza con mucha frecuencia.

Si atendemos al uso de cada una de las aplicaciones de Google Drive la más utilizada, ya sea en solitario o en compañía de cualquiera de las otras funcionalidades, es la destinada a crear, editar y compartir documentos de texto: el 77,08\% de los alumnos y alumnas que manifiestan haber utilizado Google Drive afirman haber usado esta utilidad.

De entre las 3 aplicaciones asimilables a cualquier suite de ofimática (tratamiento de textos, hoja de cálculo, presentaciones) la menos utilizada es la hoja de cálculo -un 12\% de alumnos, de entre los que han utilizado Google Drive, dicen haberla usado-.

\subsection{Descripción y análisis de la utilidad del aprendizaje y uso de Google Drive}

Dentro de los alumnos y alumnas que afirman haber utilizado Google Drive para trabajar en equipo, el 64,79\% lo considera como una herramienta muy útil. El $32,75 \%$ piensa que es útil y sólo un 2,46\% considera que Google Drive no es útil para trabajar cooperando.

Parece importante subrayar dos aspectos que se consideran interesantes:

- El 100\% del grupo de Aprendizaje Cooperativo, que trabaja de modo sistemático con la herramienta, la considera muy útil.

- El 100\% de los individuos que no encuentran útil Google Drive cursan bachillerato.

Por otra parte, si se analizan de manera conjunta, se aprecia que la valoración que los sujetos hacen de la utilidad de Google Drive como herramienta de trabajo cooperativo y la consideración sobre la utilidad de su aprendizaje guardan una relación directa. Se plasman los datos en el Gráfico 10.

Un 96,64\% recomiendan el aprendizaje de Google Drive de los cuales:

- Un $74,31 \%$ lo consideran útil tanto ahora como en el futuro.

- Un 12,50\% lo consideran útil sólo ahora, considerando que no será de utilidad en el futuro. No obstante, a pesar de esta objeción, sólo el 5,26\% no recomiendan el aprendizaje de Google Drive.

- Un $12,15 \%$ consideran que Google Drive no es útil ahora pero sí lo será en el futuro. Aunque menos incongruente que en el caso anterior, sigue siendo llamativo que sólo el 10,26\% de estos sujetos no recomiendan aprender a utilizar Google Drive. 
MIGUEL ÁLVAREZ FERRÓN Y LUIS SÁNCHEZ CAÑIZARES

CONOCIMIENTO, VALORACIÓN Y UTILIZACIÓN, POR PARTE DEL ALUMNADO, DE GOOGLE DRIVE COMO HERRAMIENTA DE TRABAJO COOPERATIVO

\section{GRÁFICO 10}

Comparación utilidad Google Drive como herramienta de trabajo cooperativo y utilidad de su aprendizaje

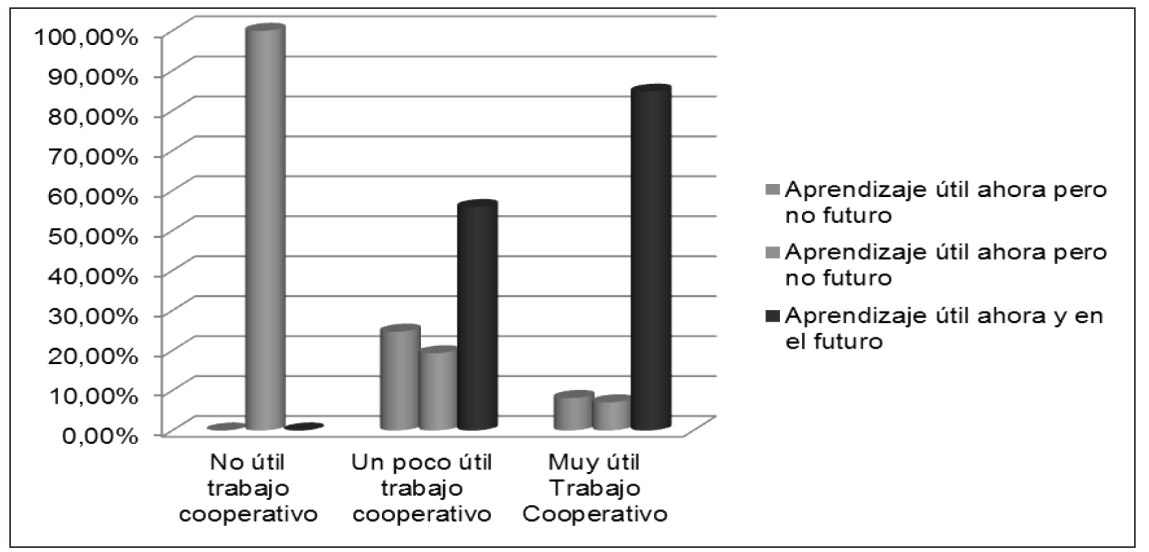

\subsection{Análisis del uso previo de Google Drive y dificultad de aprendizaje}

En cuanto al uso previo de Google Drive:

- Un $70,45 \%$ ha aprendido a utilizar Google Drive en el instituto. De ellos, el 36,24\% reconoce que nunca había utilizado Google Drive. Un 34,21\% ha expresado que lo había usado en alguna ocasión pero que aprendió a utilizarlo en el instituto.

- Un 29,55\% manifiesta que sabía utilizarlo y de hecho lo usaba antes de acudir al instituto.

GRÁFICO 11

Opinión sobre dificultad de aprendizaje de Google Drive

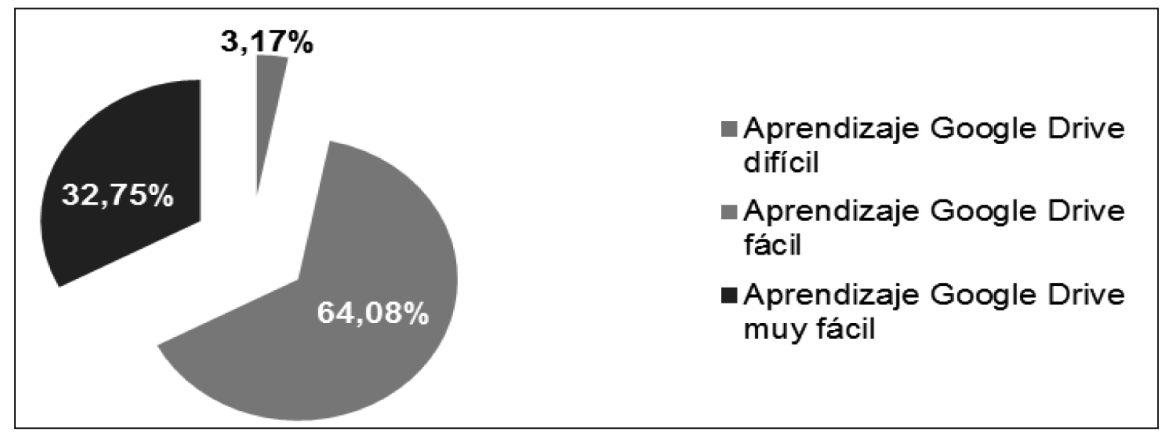


MIGUEL ÁLVAREZ FERRÓN Y LUIS SÁNCHEZ CAÑIZARES

CONOCIMIENTO, VALORACIÓN Y UTILIZACIÓN, POR PARTE DEL ALUMNADO, DE GOOGLE DRIVE COMO HERRAMIENTA DE TRABAJO COOPERATIVO

Si se refiere la opinión de los estudiantes sobre la dificultad que entraña aprender a utilizar Google Drive resulta que casi un 97\% considera fácil o muy fácil aprender a utilizar Google Drive (Gráfico 11).

El 100\% de los que consideran que aprender a utilizar Google Drive no ha sido sencillo, pese a esa dificultad, recomiendan su aprendizaje.

GRÁFICO 12

Comparación uso previo de Google Drive y dificultad de aprendizaje

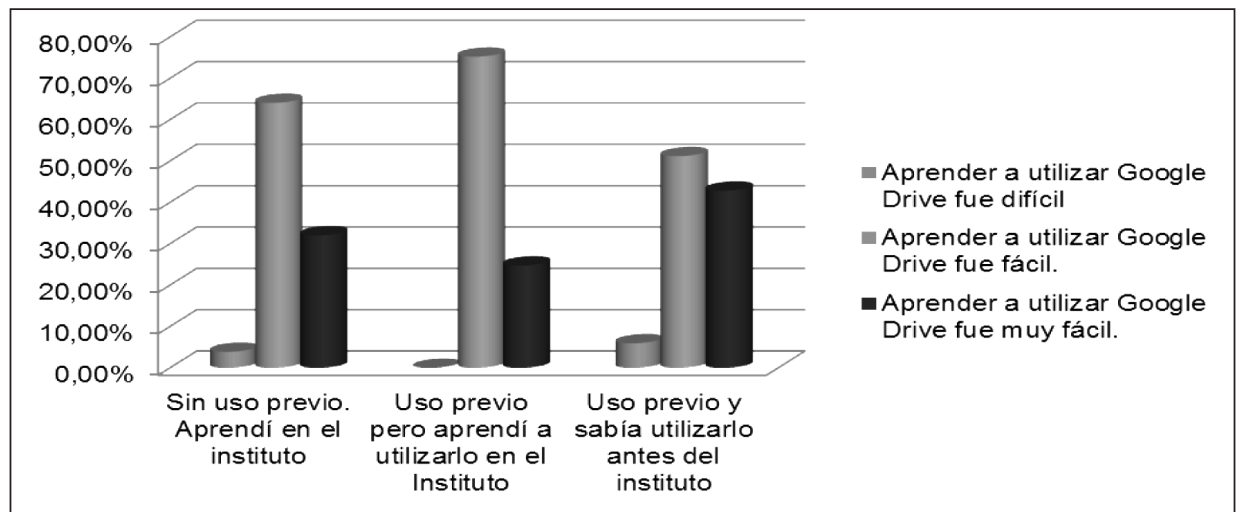

Para concluir, se analiza, en el Gráfico 12, la influencia del uso previo de Google Drive y de haber aprendido utilizarlo, o no, en el instituto en la valoración de la dificultad de aprendizaje. Del análisis resulta que ninguno de los que manifiesta tener nociones previas, pero haber aprendido a utilizar Google Drive en el instituto, considera difícil el aprendizaje. El porcentaje de los que lo considera difícil es más alto entre los que aprendieron a utilizarlo fuera del instituto.

\section{DISCUSIÓN}

Parece interesante resaltar la existencia de trabajos de investigación que guarden cierta conexión con éste, en lo que al concreto estudio de Google Drive se refiere. Entre ellos se pueden destacar algunos como los de Delgado Benito et al. (2011 y 2012), García-Vázquez et al. (2012) o Miranda (2008).

A la vista de los estudios con los que ha trabajado se puede concluir lo siguiente:

- La mayoría de los estudios sobre el uso académico de Google Drive o su "antecesor" Google Docs se caracterizan por referirse, a diferencia de este estudio, a concretas experiencias que suponen la puesta en práctica de un método de trabajo cooperativo utilizando esta aplicación de Google como herramienta para trabajar cooperando. 
MIGUEL ÁLVAREZ FERRÓN Y LUIS SÁNCHEZ CAÑIZARES

CONOCIMIENTO, VALORACIÓN Y UTILIZACIÓN, POR PARTE DEL ALUMNADO, DE GOOGLE DRIVE

COMO HERRAMIENTA DE TRABAJO COOPERATIVO

- Aunque existen más trabajos sobre experiencias en educación infantil y en estudios superiores, también hay un número significativo de estudios referidos a Educación Secundaria o Formación Profesional. No obstante, la experiencia de trabajo cooperativo con Google Drive se suele estudiar desde el prisma de su aplicación a una única asignatura.

- Los estudios valoran de una forma positiva Google Drive como herramienta de trabajo cooperativo en educación.

- Todos constatan una mejora de los resultados, lo que está fuera del ámbito de los objetivos de este estudio.

- Manifiestan, además, que se trata de aplicaciones útiles y que mejoran la motivación del alumnado. El presente trabajo viene a confirmar estas afirmaciones desde la óptica del alumnado.

\section{CONCLUSIONES Y REFLEXIÓN PERSONAL}

\subsection{Conclusiones}

Primera. La muestra del IES Príncipe de Asturias de Lorca manifiesta realizar actividades académicas cooperando entre ellos en un porcentaje cercano al 100\%. Si bien no es trabajo cooperativo en sentido estricto, no parece hiperbólico aventurar, si además se tiene en cuenta lo que se va a expresar en la conclusión segunda, que se trata de un terreno roturado y abonado para la utilización de ese método de enseñanza-aprendizaje.

Segunda. Casi el 90\% considera útil la realización de trabajo cooperativo. Dentro del mismo existe una porción, un $28,80 \%$ del total, que sólo percibe la ventaja de realizar los trabajos con mayor rapidez, considerando que el hecho de trabajar cooperando no influye en el aprendizaje. No obstante, es mayoritario el porcentaje $-59,71 \%$ sobre el total de la muestra- que considera que el hecho de trabajar cooperando redunda en una mayor facilidad para el aprendizaje.

Tercera. El alumnado manifiesta, en un porcentaje superior al 70\%, que utiliza las TIC de manera habitual o muy habitual como herramienta para trabajar cooperando con sus compañeros. El uso puntual se sitúa en torno al 25\% y sólo una porción inferior al 3\% de la muestra afirma no utilizar las TIC para trabajar en equipo con sus compañeros/as. Los dispositivos más utilizados para el acceso a las TIC son el ordenador personal y el teléfono móvil, ya sea de forma independiente o por separado. Las redes sociales, el correo electrónico y los servicios de mensajería instantánea como Whatsapp son los medios que el alumnado dice utilizar mayoritariamente para trabajar en equipo con sus compañeros. Entre los que conocen Google Drive su utilización como herramienta para realizar trabajos cooperando supera el $90 \%$.

Cuarta. El porcentaje de la muestra que considera que el uso de TIC no es útil para trabajar cooperando, o que piensa que éstas influyen de manera negativa en la motivación, se sitúa alrededor del $2 \%$. La mayoría del alumnado valora estos 
MIGUEL ÁLVAREZ FERRÓN Y LUIS SÁNCHEZ CAÑIZARES

CONOCIMIENTO, VALORACIÓN Y UTILIZACIÓN, POR PARTE DEL ALUMNADO, DE GOOGLE DRIVE COMO HERRAMIENTA DE TRABAJO COOPERATIVO

dos parámetros de forma positiva. La relación existente entre los mismos es de proporción directa. También es directamente proporcional el uso de las TIC, tanto con la valoración de su utilidad, como con la opinión sobre su influencia en la motivación

Quinta. Más del 50\% de la muestra manifiesta no conocer Google Drive. Sólo existe un nivel alto de conocimiento del mismo en el grupo que trabaja con este producto de manera sistemática. Además el conocimiento y uso del producto es muy fragmentado.

Existe una relación de proporcionalidad directa entre el uso de Google Drive y la consideración de la dificultad de su aprendizaje.

Sexta. Más de un 97\% de los alumnos que utilizan Google Drive lo consideran útil/muy útil como herramienta para realizar trabajo cooperativo. El porcentaje de alumnos que recomienda su aprendizaje supera el 95\%. Entre los que cuestionan de manera parcial su utilidad -ya sea presente o futura- el porcentaje de estudiantes que no recomienda su aprendizaje alcanza el 10\%. El 100\% del grupo que trabaja de forma sistemática con la aplicación la considera muy útil y la recomienda. La relación entre las variables utilidad y recomendación de Google Drive es de proporcionalidad directa.

La apreciación de la dificultad de aprendizaje no afecta a la recomendación de Google Drive.

\subsection{Reflexión personal. Propuesta de mejora}

Una vez concluido el estudio en todas sus vertientes, tanto la empírica como la opinión doctrinal, lo que resulta más llamativo es el desconocimiento de Google Drive y su poco uso "absoluto". Si se atiende a su uso "relativo" (por quien lo conoce) el porcentaje de utilización se sitúa por encima del 90\%, guardando una relación de proporcionalidad directa con la opinión favorable sobre su utilización.

A nuestro juicio y a la vista de:

- La valoración positiva que el alumnado tiene sobre el trabajo en equipo.

- La posesión por parte de los alumnos de una competencia digital y de la opinión favorable al uso de las TIC, por considerarlas útiles y motivadoras.

- La disposición de un software, aunque de funcionalidad limitada, gratuito, que facilita el uso de una metodología cooperativa, cuya influencia positiva en el proceso de enseñanza-aprendizaje es manifestada de modo casi unánime por la doctrina.

- Se trata de un software que no sólo permite cooperar, sino que su utilización dotaría al alumnado de competencias digitales en tecnologías de la información. 
MIGUEL ÁLVAREZ FERRÓN Y LUIS SÁNCHEZ CAÑIZARES

CONOCIMIENTO, VALORACIÓN Y UTILIZACIÓN, POR PARTE DEL ALUMNADO, DE GOOGLE DRIVE

COMO HERRAMIENTA DE TRABAJO COOPERATIVO

Su uso con el alumnado podría suponer facilitarle una herramienta que no sólo incrementaría su competencia digital, sino que, sobre todo, pondría a su disposición un salmer robusto sobre el que descanse el proceso constructivo de su aprendizaje. Dicho lo anterior con las debidas precauciones, que se deberían asumir, para afrontar los aspectos negativos de Google Drive-sobre todo los referidos a la seguridad y protección de la privacidad- y entendiendo las virtudes del uso de esta aplicación pueden ser extrapoladas a cualesquiera otras propias de la filosofía web 2.0, o incluso utilizando otras plataformas could computing asociadas al uso de un paquete ofimático.

\section{REFERENCIAS BIBLIOGRÁFICAS}

Anderson, P. (2007). What is Web 2.0? Ideas, technologies and implications for education. JISC Technology and Standards Watch. Descargado el 14 de marzo de 2014. www.jisc.ac.uk/media/documents/.../tsw0701b.pdf.

Cadoche y cols. Reglas para la elaboración de cuestionarios. Descargado el 14 de marzo de 2014.

http://www.rrppnet.com.ar/comohacerunaencuesta.htm

Castañeda Quintero, L. (2007). Software social para la escuela 2.0: más allá de los Blogs y las Wikis. En Inclusión Digital en la Educación Superior: Desafíos y Oportunidades en la sociedad de la Información. X Congreso Internacional EDUTEC. Edición electrónica. Buenos Aires 5 .

Delgado Benito, V. y Casado Muñoz, R. (2012). Google Docs: una experiencia de trabajo colaborativo en la Universidad. Enseñanza \& Teaching: Revista Interuniversitaria de Didáctica, 30 (1), 159-180.

Delgado Benito, V. et al. (2011). Google Docs una experiencia de trabajo colaborativo desde las aulas universitarias. En A. Hernández Martín y S. Olmos Migueláñez (Eds.). Metodologías de aprendizaje colaborativo a través de las tecnologías (pp. 328-338). Salamanca, Ediciones Universidad de Salamanca.

Díaz González, E. M. y Piñana López, J. A. (2013). Material de cátedra de la asignatura "Innovación Educativa”. Máster Universitario en Formación del Profesorado, Universidad de Murcia.

García-Vázquez, C. C. y Tejera Arcenillas, I. (2012). Resultados de la utilización de Google Docs en procesos de mentorización con alumnado de tercer ciclo. Trabajo: Revista Andaluza de Relaciones Laborales, 25-26, 135-153.

Gutiérrez Gallardo, J. D. y López Guisado, A. (2007). Google. Guía práctica para usuarios. Madrid: Anaya.

Johnson, D. W.; Johnson, R. T. y Holubec, E. J. (1999). El aprendizaje cooperativo en el aula. Traducción de Gloria Vitale. Buenos Aires: Paidós.

O'Reilly, T. (2005). What is Web 2.0: Design Patterns and Business Models for the next generation of software. Descargado el 14 de marzo de 2014.

http://oreilly.com/web2/archive/what-is-web-20.htm.

5. Texto sin paginar. 
Pujolás Maset, P. (2004). Aprender juntos alumnos diferentes. Los equipos de aprendizaje cooperativo en el aula. Barcelona: Octaedro.

Scagnoli, N. I. (2005). Estrategias para motivar el aprendizaje cooperativo en cursos a distancia. Urbana-Champaign, Illinois. Descargado el 14 de marzo de 2014. http://hdl.handle.net/2142/10681.

Suárez Guerrero, C. (2010). Cooperación como condición social de aprendizaje. Barcelona: UOC.

Trujillo Sáez, F. y Ariza Pérez, M. A. (2006). Experiencias educativas en aprendizaje cooperativo. Granada: Grupo Editorial Universitario.

Uriz Bidegáin, N. et al. (1999). El aprendizaje cooperativo. Pamplona: Gobierno de Navarra. 